\title{
les caractéristiques mécaniques des sols fins déduites des essais d'identification
}

\author{
par \\ J. Hurtado \\ Ingénieur Civil des Ponts et Chaussées \\ Directeur-Gérant de "Sols et Structures"
}

\begin{abstract}
L'usage d'abaques ou de formules de corrélation est très répandu en mécanique des sois. Les essais in situ ont largement bénéficié de cet état d'esprit. En revanche, les essais d'identification font rarement l'objet d'une utilisation directe. Les travaux sur ce sujet de SKEMPTON en Angleterre et de BUISSON et de BIAREZ en France n'ont pas connu le succès qu'ils méritaient dans la pratique courante.

L'abaque que nous présentons permet de déduire des limites d'Atterberg la droite œdométrique. L'indice des vides d'un échantillon intact permet alors de connaître la courbe œdométrique complète et les caractéristiques mécaniques du sol en place. On peut en outre suivre l'évolution des caractéristiques mécaniques du sol en cours de consolidation.
\end{abstract}

\section{Limites d'Atterberg}

Les essais d'Atterberg sont peu coûteux et peuvent être facilement réalisés sur des échantillons remaniés. Les résultats sont très fiables: on obtient, pour un même sol confié à des laboratoires différents, des résultats très concordants à un ou deux points près.

L'abaque que nous présentons comporte deux courbes appelées «trace de liquidité" et "trace de plasticité $\%$.

La droite cedométrique est obtenue de la façon suivante :

— On calcule l'indice des vides à la limite de liquidité

$$
e_{L L}=\frac{w_{L L}}{100} \cdot \frac{\gamma_{s}}{\gamma_{w}}
$$

On porte le point A d'ordonnée $e_{\mathrm{LL}}$ sur la * trace de liquidité ».

- On calcule l'indice des vides à la limite de plasticité

$$
e_{L P}=\frac{w_{L P}}{100} \cdot \frac{\gamma_{S}}{\gamma_{w}}
$$

On porte le point $\mathrm{B}$ d'ordonnée $\mathrm{e}_{\mathrm{LP}}$ sur la «trace de plasticitén.
La droite $\mathrm{AB}$ est la droite œdométrique.

\section{Indice des vides naturels}

On mesure l'indice des vides e d'un échantillon intact d'un sol dont on a par ailleurs mesuré les limites d'Atterberg et tracé la droite $A B$.

On porte sur l'axe des a e $»$ le point $C$ d'ordonnée e.

On porte sur $A B$ le point $P$ d'ordonnée e.

On porte sur $\mathrm{PB}$ le point $\mathrm{D}$ tel que $\mathrm{CP}=\mathrm{PD}$.

On trace l'arc de cercle $C D$ tangent à $C P$ et $P D$.

La courbe codométrique est $\mathrm{CDB}$.

On porte sur l'arc CD le point M d'abscisse CP.

$M$ est le point représentatif du sol en place. On lit sur les familles de courbes correspondantes $C_{u}$ et $\varphi^{\prime}$.

Si l'on étudie la consolidation d'un sol mou l'abaque permet de suivre le déplacement du point représentatif $M$ en cours de consolidation et de connaître les futures caractéristiques plastiques $C_{u}$ et $\varphi^{\prime}$ du sol consolidé.

\section{Domaine d'application}

On ne peut utiliser l'abaque que pour des sols peu sensibles et non cimentés, et tels que $15<I_{p}<100$ et de consistance $0<I_{c}<1$.

\section{Précision}

Les tassements calculés avec cet abaque sont compris entre 0,9 et 1,3 fois les tassements calculés à partir de la courbe œdométrique réelile.

Les caractéristiques mécaniques données par l'abaque sont comprises entre 0,8 et 1,2 fois les caractéristiques obtenues par l'essai triaxial. 


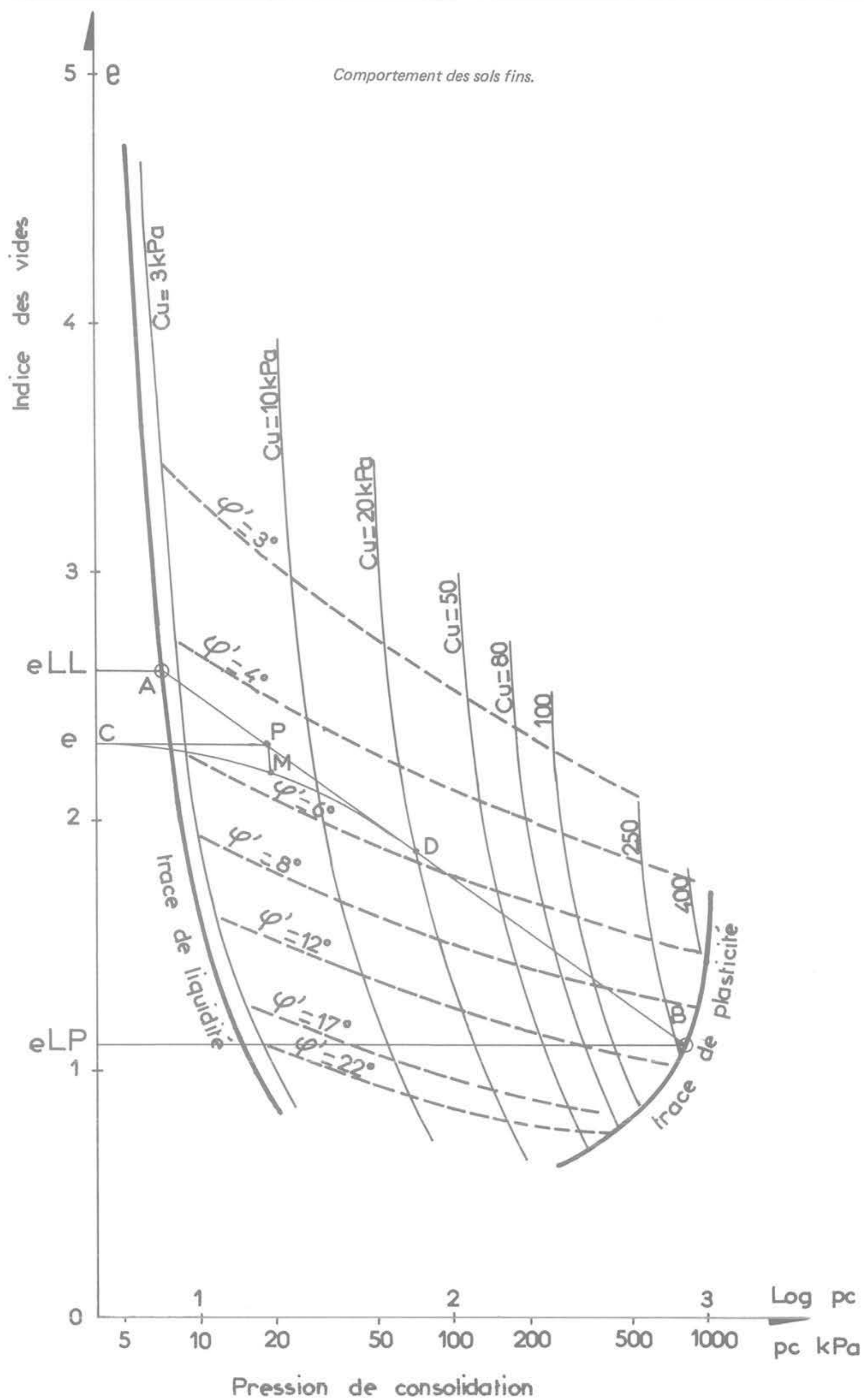

